\title{
Last Value Prior to Dosing Imputation Technique
}

National Cancer Institute

\section{Source}

National Cancer Institute. Last Value Prior to Dosing Imputation Technique. NCI

Thesaurus. Code C132342.

A data imputation technique that populates missing values with the subject's last observed value prior to the start of study treatment. 